University of Warwick institutional repository: http://go.warwick.ac.uk/wrap This paper is made available online in accordance with publisher policies. Please scroll down to view the document itself. Please refer to the repository record for this item and our policy information available from the repository home page for further information.

To see the final version of this paper please visit the publisher's website. Access to the published version may require a subscription.

Author(s): David Tall and Mohamad Rashidi Razali

Article Title: Diagnosing students' difficulties in learning mathematics

Year of publication: 1993

Link to published version: http://dx.doi.org/ doi:

10.1080/0020739930240206

Publisher statement: None 


\title{
Diagnosing Students' Difficulties in Learning Mathematics
}

\author{
David Tall \\ Department of Science Education, \\ University of Warwick, \\ Coventry CV4 7AL \\ United Kingdom. \\ Mohamad Rashidi Razali \\ Department of Mathematics, \\ Universiti Teknologi Malaysia, \\ Locked Bag No. 791, \\ 80990 Johor Bahru \\ Malaysia
}

\section{Introduction}

The aim of mathematical education is surely success for all pupils, yet it seems to be a fact of life that whilst a few prosper in mathematics, a much greater number find mathematics difficult. Thus it is that, however successful a course may appear to be, there are students who begin to struggle and who will need appropriate help to be able to pursue mathematics further.

In this paper we will discuss research into the diagnosis of the difficulties experienced by students and propose a theory which suggests a continual divergence in performance between those who succeed and those who fail, exacerbated by qualitative differences in their thinking processes. Gray and Tall (1991) give evidence of younger children performing arithmetic which shows that when taught arithmetic procedures, the less able seek security in carrying out the process whilst the more able soon develop a more flexible kind of thinking in which notation is used to mean either a process to give a result or a concept to be manipulated at a higher level. A procept is defined to be the amalgam of process and concept which is represented by the same symbolism, thus $2+3$ represents the process of addition of 2 and 3, and the result of the process (5), $2+3 x$ represents both the process of adding 2 to 3 times $x$ and the resulting expression, $\sin x=$ opposite/hypotenuse represents both the process of calculating the sine as a ratio of lengths and also the value of the sine as the result of the calculation. It is hypothesised that less successful students have greater difficulties with the processes and concentrate on the lesser target of being able to carry them out satisfactorily whilst more successful students develop a more flexible method of handling notation, being able to see it either as a process to calculate a needed result or as an object to be manipulated as part of a more complex piece of symbolism. In other words success 
comes from flexible proceptual thinking whilst short-term success but long-term failure comes from procedural thinking.

Here we are concerned with the performance of students transferring to the Universiti Teknologi Malaysia at 16+ who find difficulty with the mathematics that is pre-requisite for courses in engineering and other sciences. The students attending this university are in the 50th to 90th percentile of the ability range for the whole population and therefore represent a broader ability band than that normally attending university in many countries. It therefore increases the perceived gap in ability between the most and least able and, if there is a qualitative difference in thinking processes to be observed, then it is likely to be seen here.

In sections 2 and 3 we give a brief background to the study and the design of a diagnostic test to reveal student difficulties. In section 4 we look at examples of the errors which arise and in section 5 we consider how these errors might arise from the hypothesised qualitative difference in thinking processes. Section 6 considers the implications of this theory and section 7 describes how a remedial course is being designed to take account of these ideas.

\section{Background to the research}

A research group called Diagnostic and Remedial Mathematics Group was formed at the Universiti Teknologi Malaysia in September 25, 1987 with the primary task of identifying the difficulties encountered by students and then to use this information to develop remedial procedures to overcome these difficulties.

At first the group considered various methods of diagnosing difficulties. For instance, they considered interview techniques, such as getting the students to think aloud (Newell \& Simon, 1972; Bartlett, 1954), with students being asked to verbalize every thought that comes to their mind in the course of solving a mathematics problem. A variant of this technique, developed originally by Jean Piaget, is the clinical interview (Af Ekenstam \& Greger, 1983; Greer, 1987). This differs from "thinking aloud" in that the teacher may follow the thoughts of his students by asking questions here and there during the course of solving the particular problem.

Interviews with students, whilst giving subtle indications of the existence of cognitive difficulties, need to be complemented by broader studies which indicate the extent of such difficulties in the population under consideration. Therefore the Diagnostic and Remedial Group planned to develop a test paper consisting of multiple choice questions whose design was based on suspected conceptual difficulties with questions formulated 
to induce conceptual errors. They were aware that the errors encountered might come from a number of sources, including sensory, mental, emotional, motivational, cultural, social, reading or instructional deficiencies (Bell, 1978). They were also aware of difficulties known at school level which have yet to be properly diagnosed and remediated, as reported by the Malaysian Ministry of Education (see the Cabinet report on Education, 1979, pp.18-20) and the report by the Ministry of Education (1982, p. 18).

A preliminary mathematics diagnostic investigation was given to 350 first year students in August 1988, using questions requiring a written response which were designed to provoke possible misunderstandings and errors. The first multiple-choice diagnostic test was then designed using evidence from the preliminary investigation to construct problems that may lead to observed errors. This Mathematics Diagnostic Test was implemented to the first year students in October 1989. It proved highly successful in discriminating between students of different capabilities and has since been partially modified for a test in July 1990 by replacing, or modifying, those questions which were less successful discriminators.

\section{Overall Design of the Diagnostic Test}

For the purposes of analysis, two groups of students were identified from those taking the diploma, where difficulties were more likely to occur. These were those in the highest 27\% (group H) and those in the lowest 27\% (group L). For each of the forty questions on the diagnostic test, the proportion of correct responses was calculated for groups $\mathrm{H}$ and $\mathrm{L}$. The average of these correct responses gives a number (called the $P$ index) which is a measure of the probability that a given student will obtain a correct answer; the difference between the proportions for the groups $\mathrm{H}$ and $\mathrm{L}$ is an indication of the extent that the question discriminates between the high and low performers (called the $D$ index). Questions with a $P$ index of about 1 would be too easy, because nearly everyone would get them right, and questions with a $P$ index of around 0 would be too hard because almost no-one would be able to do them. "Good" questions would have a $P$ index of around 0.5 and a suitably high $D$ index (indicating a high discrimination between those of high and low performance).

Overall the October 1989 Diagnostic Test proved to be an excellent instrument for diagnosis. Only seven questions out of 40 had $D \leq 0.25$. Of these two had a low $P$ index and were too difficult; the other five had a high $P$ index and were too easy. Thirty three questions out of forty were good discriminators. Some of the effort of redesigning the test was addressed to modifying those which were not such good 
discriminators, however, the results of the second test were very similar to the first and so the remainder of this article considers the 1989 version.

\subsection{Analysis of error types}

An overall analysis of errors was performed by considering the wrong choices made on the multi-choice questions. Two types of scenario were distinguished:

(1) The "hard" questions which had a high proportion of errors in both the $\mathrm{L}$ and $\mathrm{H}$ groups,

(2) The "discriminating" questions where the H group did well and the L group obtained less than $50 \%$ correct responses.

Type (1) was indicative either of a peculiarly difficult question or of a problem which applied to almost all students. Type (2) indicated areas where the more able were generally successful but the less able were making significant errors. The former requires overall remediation and perhaps reconsideration of the earlier teaching methods, the latter requires more specific remediation for individuals who fail to cope with topics which have been better understood by the more able.

An initial scan of the errors reveals a wide array of difficulties with specific knowledge. However, the nature of the process failures followed a much smaller number of different patterns. We hypothesise that these patterns are indicative of a qualitative difference in the thinking between more and less able students. It is essential in both diagnosis and the design of remediation to be aware of these differences.

\section{Examples of errors}

Errors arising on the diagnostic test suggested underlying differences in the thinking processes of the students. Here we give a few selective examples from the 40 questions on the test.

\section{Order of precedence of arithmetic operations (question 4)}

$$
(P=0.5, \quad \mathrm{D}=0.4)
$$

When faced with the choice:

$$
2 \times 6-4+40 \div 2=\left\{\begin{array}{l}
\text { A. } 22 \\
\text { B. } 24 \\
\text { C. } 28 \\
\text { D. } 42 \\
\text { E. other }
\end{array}\right.
$$


a majority $(61 \%)$ of group $L$ responded by asserting that $2 \times 6-4+40 \div 2=24$, which indicates reading left to right with no regard to the precedence of multiplication. This is the way that the calculation is carried out on some calculators. It indicates a treatment of the arithmetic symbols as a sequence of operations to be carried out in the order that they are read. Meanwhile a majority of group $\mathrm{H}$ scanned the symbols and gave the answer 28 which indicates that they have understood the order of precedence of operators. Thus one may hypothesise that the less able see the symbols as a process to be carried out in the written order, whilst the more able can chunk the symbols together as sub-expressions to be carried out according to given conventions.

The meaning of $2 \log _{10} 5+\log _{10} 8-\log _{10} 2$ (question 5 )

$(P=0.62, D=0.51)$

The problem

$$
2 \log _{10} 5+\log _{10} 8-\log _{10} 2=\left\{\begin{array}{l}
\text { A. } \log _{10} 16 \\
\text { B. } \log _{10} 31 \\
\text { C. } 2 \\
\text { D. } 100 \\
\text { E. other }
\end{array},\right.
$$

presents the students again with the problem of processing a group of symbols. Students who see the symbols as representing a process to carry out will have to conceive this as multiplying the logarithm (to base 10) of 5 by 2 , then adding the logarithm of 8 and subtracting the logarithm of 2 . This is a long sequence of operations which is complicated to coordinate, yet if the student can chunk it into sub-expressions and knows the properties of logarithms, then $2 \log _{10} 5$ may be seen as $\log _{10}\left(5^{2}\right)=\log _{10} 25$, and then the three sub-expressions combined as

$$
\log _{10} 25+\log _{10} 8-\log _{10} 2=\log _{10}(25 \times 8 / 2)=\log _{10}(100)=2 .
$$

The $D$ index of 0.51 shows that group $\mathrm{L}$ are far less adept than group $\mathrm{H}$ at the chunking necessary to give the correct answer.

Manipulating algebraic equations (Question 15) $\quad(P=0.66, D=0.51)$

Group L had some difficulty with the question: 
If $k=\frac{r}{r-p}$, then $r$ in terms of $p$ and $k$ is $\left\{\begin{array}{l}\text { A. } \frac{r+k p}{k} \\ \text { B. } \frac{k p}{k-1} \\ \text { C. } k r-k p \\ \text { D. } \frac{k p}{1-k} \\ \text { E. other }\end{array}\right.$

Algebra is seen as a difficult subject for which the traditional cure when a student fails is lots of practice exercises. However, we would contend that such exercises may only serve to prolong the misinterpretation that algebra is a menagerie of disconnected rules to deal with different contexts ("collect together like terms", "turn upside down and multiply", "do the same thing to both sides", "change side, change sign", etc, etc). Tall $\&$ Thomas (1991) suggest that there are several cognitive obstacles in the early learning of algebra, for instance the fact that an expression is seen as a process to be carried out $(2 x+3$ is "multiply $x$ by 2 and add 3") and not as an object which can be manipulated. Since the less able are often not willing to accept such an expression as meaningful (because they cannot work it out until they know the value of $x$ and, if they know the value of $x$ they needn't do algebra, they could do arithmetic), they are uneasy about handling it and sink into the use of (ill-)remembered procedures. Here group L are less secure than group $\mathrm{H}$ in carrying out the sequence of manipulations necessary to give the correct result.

Solve $(x-2)(x+3)=6 x$ (question 10)

$(P=0.7, D=0.48)$

An interesting, and not unexpected, difficulty occurs with the following:

$$
\text { If }(x-2)(x+3)=6 x \text {, then the values of } x \text { are }\left\{\begin{array}{l}
\text { A. }-6,1 \\
\text { B. } 6,-1 \\
\text { C. }-2,-3 \\
\text { D. } 2,3 \\
\text { E. other }
\end{array}\right.
$$

A likely difficulty with the L group is that they may evoke (incorrectly) part of the process of solving equations in that the left hand side is factorised. This acts as a distractor and may lead to the selection of one of the incorrect solutions. Once more Group L seem to have problems with the meaning of the symbolism, evoking part of the procedure to solve equations by factorisation and, being unable to coordinate the procedure correctly, fall into error. If students are unable to give appropriate meaning, then procedures are likely to be carried out by rote and highly prone to failure. Tall $\&$ Thomas (1991) have shown how a blend of BASIC programming, computer software to give meaning to algebraic expressions and games to carry out analogous procedures 
to those of a computer manipulating variable stores, can lead to more versatile understanding and flexible use of algebraic notation.

The singular case of simplifying $\frac{x-1}{2-x}-\frac{1}{x-2}$ (question 12)

$(P=0.62, D=0.43)$

Processes that follow a general pattern yet which have certain singular cases that behave differently cause great problems to weaker students. They try to operate the general procedure and cannot cope with the singular difficulty. A case in point occurs in the following question:

Simplify $\frac{x-1}{2-x}-\frac{1}{x-2}$ :
A. $-\frac{1}{2} x$
B. $\frac{x}{2-x}$
C. $\frac{x}{x-2}$
D. $\frac{x^{2}-4 x}{(2-x)(x-2)}$
E. other.

Here the two denominators are essential the same, except for a minus sign. Yet less successful students may not see this and try to use the product of $2-x$ and $x-2$ as the common denominator during simplification. Only $6 \%$ of group $\mathrm{L}$ gave the correct answer (B). The $45 \%$ of group L giving the distractor $\frac{x^{2}-4 x}{(2-x)(x-2)}$ shows the desire to follow the "general method" to find the lowest common denominator.

Such singular cases will usually cause problems. (Another is to solve $a x=a$ for $x$, which has a singular case when $a=0$.) They require specific teaching to show how the singular cases fit in the general pattern. In this case it requires revision of the process of handling rational expressions and, within this process, to deal with singular cases which may not appear at first sight to fit the pattern.

The modulus sign (question 2)

$(P=0.69, D=0.46)$

The modulus sign is known to pose difficulties to students (Chiarugi et al, 1990). Many students have difficulty in thinking of $|x|$ as $-x$ when $x$ is negative, probably because of the difficulty of conceiving of something starting with a minus sign as being positive. This leads to difficulty in manipulating expressions involving the modulus sign as in the question: 


$$
\text { If } x=1 \text { and } y=4 \text {, then }|x-y|=\left\{\begin{array}{l}
\text { A. }-3 \\
\text { B. } \frac{1}{4} \\
\text { C. } 3 \\
\text { D. } \sqrt{17} \\
\text { E. other }
\end{array}\right.
$$

A significant minority in group L say

$$
|x-y|=-3 \text {. }
$$

The meaning of the symbolism is plainly unclear to these students, and it may be made worse by the difficulty coordinating two processes, here the action of subtraction and the taking of the modulus.

\section{The inverse function (question 7)}

Functions give students great difficulty (Markovits et al 1988, Vinner 1983, Tall \& Bakar, to appear). The inverse function causes even more problems, as witnessed by responses to the following:

A function $\mathrm{f}$ is defined by $\mathrm{f}: x \rightarrow \frac{x-1}{x-2}, x \neq 2$.

The expression $\mathrm{f}^{-1}$ in a similar form is
A. $\mathrm{f}^{-1}: x \rightarrow \frac{1}{2}$
B. $\mathrm{f}^{-1}: x \rightarrow \frac{x-2}{x-1}$
C. $\mathrm{f}^{-1}: x \rightarrow \frac{2 x-1}{1-x}$
D. $\mathrm{f}^{-1}: x \rightarrow \frac{2 x-1}{x-1}$
E. other.

A high $D$ index shows the wide difference in performance between groups $\mathrm{H}$ and $\mathrm{L}$. Professional mathematicians often misunderstand the notion of inverse, so we should not be surprised by the difficulties of students. The procedural thinker is faced with the difficulty of undoing a process. A function $y=\mathrm{f}(x)$ is a process: input $x$, carry out a procedure, output $y$. The reverse process: what $x$ must be put in to get $y$ out is much harder. If the function is not be one-one then there may be several $y$ 's which give the same $x$, so the 'inverse function' may not be a properly defined function - unless one artificially restricts its domain or allows the inverse to give a set of pre-images. For instance, if $\mathrm{f}(x)=|x|$, what is $\mathrm{f}^{-1}(x)$ ? In the case of $\mathrm{f}(x)=x^{5}-17 x^{3}+4 x^{2}-1$, finding $\mathrm{f}^{-1}(0)$ is hard, let alone $\mathrm{f}^{-1}(x)$ for general $x$ ! 
When such things are not fully understood, it is only to be expected that security will be sought in carrying out the process mechanically. In this case it might be performed by algebraic manipulation. Put $y=\frac{x-1}{x-2}$ and solve for $y$ in terms of $x$. This gives $y x-2 y=x-1$ so $x(y-1)=2 y-1$, so $x=\frac{2 y-1}{y-1}$. This is $\mathrm{f}^{-1}(y)$. Hence (changing variable!), $\mathrm{f}^{-1}(x)=\frac{2 x-1}{x-1}$.

However, we have seen earlier that the algebraic manipulation is already hard for the less able, and coordinating and reversing the procedures in this complicated way is compounding the difficulty. It is therefore no wonder that group $\mathrm{L}$ are so much more likely to fail ...

\section{Given the circumference: to find the radius (question 11)}

$$
(P=0.55, D=0.46)
$$

Students find difficulties when asked to link visual and arithmetic processes in a way which is not in the same order as normally encountered. For instance, in the following diagram, where information is given about the circumference, rather than the radius.

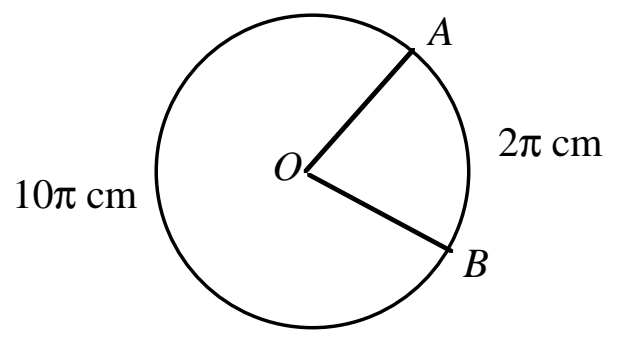

The two points $A$ and $B$ are on the circle centre $O$, the $\operatorname{arc} A B$ is $2 \pi \mathrm{cm}$, and the large arc from $A$ to $B$ is $10 \pi \mathrm{cm}$. Students are asked to say whether the radius of the circle is:
A. $\sqrt{10} \mathrm{~cm}$
B. $\sqrt{12} \mathrm{~cm}$
C. $5 \mathrm{~cm}$
D. $6 \mathrm{~cm}$
E. other.

It is highly likely that the students know the formula $c=2 \pi r$. They may fail in a number of possible ways, and the equal likelihood of three of the solutions indicates a variety of possible causes. First they may fail to properly relate different representations (graphical and algebraic). They may be unable to reverse the process (to find $r$ in terms of $c$ ). Then they may not properly coordinate the processes successfully. The lack of flexible thinking here, being unable to link the various concepts into manageable chunks, can only lead once more to greater failure for group L. 


\section{Determining the angle at the circle given the length of arcs (question 12)}

$$
(P=0.62, D=0.43)
$$

Using the same figure as question 11 , students are asked to state whether the acute angle $A O B$ is
A. $30^{\circ}$
B. $36^{\circ}$
C. $60^{\circ}$
D. $72^{\circ}$
E. other.

Again problems occur in relating representations and reversing/combining processes. The question proves to be a little easier $(P=0.62)$ but still leads to a fairly wide difference between the performances of those in groups $\mathrm{L}$ and $\mathrm{H}$.

Scale (Question 14) $\quad(P=0.44, D=0.47)$

Ratio is a known difficulty, because it relates not to an explicit number, but to relationships between pairs of numbers. Thus there is an extra level of complexity involved. On the diagnostic test the following question was given:

A map is prepared using a scale $1: 5000000$. Using this scale, a river whose actual length is $100 \mathrm{~km}$ will be represented by a length
A. $0.2 \mathrm{~cm}$
B. $0.5 \mathrm{~cm}$
C. $2 \mathrm{~cm}$
D. $5 \mathrm{~cm}$
E. other.

Group L respond by choosing each of the five alternatives in roughly the same proportion, suggesting serious misunderstandings. Here there are so many different things to coordinate: the use of appropriate mathematical symbolism, relating actual measurements to a practical graphical representation, coordinating the relationship between the lengths. It clearly requires considerable chunking of information. For instance, one way to reduce the complexity is to see that a scale 1:5000 000 is a scale factor of $\frac{1}{5000000}$ and that this must equal $\frac{x}{100}$. The solution follows by a little flexible manipulation that may be denied to the more procedural thinker who cannot cope with this degree of complexity. 


\section{Possible qualitative differences between the thinking processes of the more able and the less able.}

Having looked at specific examples from the diagnostic test, we might take the superficial view that the questions are hard and the less able students are not good enough to do them. But this is a shallow and unhelpful attitude. We must determine more succinctly what the problems are. At least we should provide a hypothesis for the differences in performance of the more able and less able.

It has been hypothesised that the objects of mathematics learning are facts and skills. The child must learn the facts and will be shown procedures (addition, multiplication, manipulation of fractions, of algebra, methods of solving equations, proofs of geometric theorems, formal calculation of derivatives, formal integration, computation of probabilities etc). So often the learner is shown how to do a procedure (preferably in a meaningful way) and then practices this until it is routinized and can be carried out (almost) automatically. Often this routine becomes crystallized into a concept. For instance, the process of counting on to calculate a sum such as $2+3$ gives the concept of the sum of two numbers, the process of formal differentiation gives the concept of derivative, and so on. It usually happens that the symbolism may indicate either or both of these, for instance $2+3 x$ represents both the process "add two to the product of three times $x$ " and also the result of the calculation, the expression " $2+3 x$ ". In this way, a dynamic process is crystallized into a static concept.

It is now more than this. The flexible thinker can switch easily from the process to the concept and back again in such a fluent way as to conceive hardly any distinction between the two. Professional mathematicians become so fluent in this ability that they often fail to distinguish explicitly between the two ways of thinking (Freudenthal, 1983, Thurston, 1990).

One finally masters an activity so perfectly that the question of how and why (students don't understand them) is not asked anymore, cannot be asked anymore and is not even understood any more as a meaningful and relevant question.

(Freudenthal, 1983, p.469)

The flexible, crystallized concept can be mentally manipulated and used for higher level thinking. For instance $2+3 x$ can be part of a more complex expression such as $(2+3 x)^{2}-15 x(2+3 x)$. The more able faced with the problem of factorisation may chunk this sub-expression as a single entity and see the factorisation

$$
(2+3 x-15 x)(2+3 x)=(2-13 x)(2+3 x) .
$$


The less able may only follow rules ... multiply out brackets, collect together like terms, look for common factors etc etc. In this way the more able is manipulating mental objects whilst the less able is having to coordinate the processes of algebra.

A major hypothesis that we propose is:

\section{The Failure in Conceptualization:}

The more able are better at crystallizing a process into a concept and so may manipulate the concept with greater fluency than the less able who may still be operating at the process level. Processes occur in time and therefore are more difficult to conceive simultaneously in the mind than concepts.

For instance, the expression

$$
2 \log _{10} 5+\log _{10} 8-\log _{10} 2
$$

which appears in the above examples from the Diagnostic Test, can be thought of either as a process or as a concept. As a process it is an instruction to multiply the logarithm of 5 by 2 , to add the logarithm of 8 and to subtract the logarithm of 2 . As a process to be carried out in time it is an arduous task whereby one may easily forget the beginning of the task as one approaches the end of it. Thus someone working at the process level will find it difficult to comprehend and hold in the mind for simplification purposes. However, seen as an object, it can be 'chunked' together as sub-objects, so that one may concentrate on the part $2 \log _{10} 5$, which equals $\log _{10} 25$, and the whole expression reduces to $\log _{10}(25 \times 8 \div 2)=\log _{10}(100)=2$.

We therefore hypothesise that the difficulties facing the less able (coordinating mental processes) are greater than those facing the more able (manipulating mental concepts). A corollary of the hypothesis of failure in conceptualization is:

The burden of the less able:

the more able are faced with conceptually easier mathematics (manipulating concepts) and so are progressively more likely to improve, whilst the less able are faced with a more difficult task (coordinating processes) and so are progressively more likely to fail.

The difference is made even greater if the more able are manipulating symbols whose relationships are in some sense meaningful to them. Meanwhile the less able are likely to be constricted within a context wherein the symbols have a more inappropriate process meaning. For instance, $(2+3) x$ and $2 x+3 x$ are totally different as processes. The first adds two and three and then multiplies the result by $x$, the second multiplies two by $x$, then multiplies three by $x$ and then adds the two results together. Thus the student with only a process interpretation will find it difficult to conceive that these two symbols represent the same thing. Meanwhile the student who sees these expressions 
as also representing the product of the two calculations will be able to focus of the fact that the results are the same and thus see the expressions as being equivalent ways of expressing the same end-result.

The more able are likely to benefit from seeing linkages and making connections between the crystallized concepts, because this new information reinforces and simplifies what they already know by placing it within a more secure knowledge structure. The more able need to remember less because they can reconstruct more. The less able see more information as a burden, as even more disjoint pieces of information to remember - an increased burden on a weaker back, leading to the greater probability of inevitable collapse.

The less able have a fundamentally different viewpoint. In addition to having less facility with mathematics, their difficulties are likely to manifest themselves in various ways, including:

(i) Being less likely to crystallize processes into manipulable concepts, thus imposing the greater burden of process coordination rather than concept manipulation,

(ii) Having fewer manipulable concepts, preferring to rely more on the security of carrying out familiar routinized processes,

(iii) Through relying on routinized processes, being less likely to relate ideas in a meaningful way.

\section{Implications of non-diagnosis of mathematical difficulties}

It is clear from this discussion that the less able students are likely to need special treatment. If this is not done, the students are likely to become progressively more confused and in the long run they may not survive in post-secondary mathematics programmes.

The literature makes some suggestions as to what may be happening. For instance, Gagné (1970) postulates that there are four phases of learning: the apprehending phase, the acquisition phase, the storage phase and the retrieval phase. This might suggest that the more able can reach these four phases but, for the less able, the last phase may be of a problem. Perhaps the mind of the less able is like a flawed computer diskette. Sometimes, it will respond well to some mathematics problems (usually the easier ones) and it will 'blank out' to more difficult ones.

But in his survey lecture at the 1988 Sixth International Congress on Mathematical Education in Budapest, Steen (1989) suggests: 
"... Teachers normally act as if each student's mind is a blank slate - or an empty computer disk - on which effective teachers can record whatever information they like. Research in cognitive science sugggests otherwise: each student's mind is more like a computer program than a computer disk. Each student brings to the mathematics classroom a rich set of prior mathematical experiences that provide a unique mental framework in which the student creates new patterns derived from new experiences. Learning occurs not in the act of remembering, but in the gradual development of mental frameworks unique to each individual. In other words, students learn by modifying their mind's program, not by storing new data in their mind's memory."

We believe that these analogies are helpful, but incomplete. They do not fully address the problem as to why the divergence between the more and less able occurs, or how the less able may be assisted to bridge the gap. We believe that the difference between the thought processes of the more able and less able leads inexorably to a growing divergence in performance in which the more able can only get comparatively better and the less able worse as the subject progresses. The more able flexibly manipulate symbols either as process or concept, whilst the less able tend to be more concerned with process and get locked into the strategy of accumulating procedures to "give the answer".

Gray \& Tall (1991) refer to the use of a common symbol to dually represent a process or a concept as a procept. Thus a procept is the amalgam of process and concept represented by the same symbol. We hypothesise that it is the ability of the more able to switch from seeing a symbol such as $2+3 x$ or $\lim _{n \rightarrow \infty} s_{n}$ as either a process or the product of that process which plays a major role in the flexibility of thought which makes them effective mathematicians. This proceptual means of thought, we claim, plays a fundamental role in causing the difference in thought between the more and less able. Whilst the more able develop a flexible proceptual method of operation, the less able are locked into an inadequate procedural method of thought which relies on coordinating an ever increasing array of disparate procedures.

\section{Proposals for remediation}

In the writing of this paper we have used the physician's metaphor of diagnosis (of weakness) and of prescription (of a cure). It is not always the most apt of metaphors. We do not believe that it is simply a case of giving the weak students the medicine which will cure them and let them re-enter the race. Instead we would see the way ahead through using our analysis of difficulty on the one hand, and the method of success of the more able on the other, to help the less able develop suitable explicit strategies that will help them be more successful. 
First the less able will clearly lack confidence. They will only gain confidence through success, so they must be helped to attain success. Simply giving them practice at the procedures which are faulty will, according to our theory, not be a solution to their problems. For their difficulty is precisely that they do not crystallize these procedures into manipulable mental objects that allows them to coordinate them to solve more complex problems.

Given the hypothesis of failure of conceptualization, less able students are more likely to operate by coordinating processes than by manipulating concepts. They already suffer from having too many separate pieces of knowledge which overburdens their memory and leads to failure. Remediation through correction of specific errors is likely to given them even more isolated pieces of information to remember, causing even greater cognitive strain and increasing the probability of failure. Furthermore, if the remedial students do not develop the more versatile form of thinking available to the more able students, then they will be ill-equipped to cope with the mathematical courses which follow and continue to suffer cognitive strain.

This suggests that such students need to focus not just on the many and varied specific errors which they have made, but on the overall powerful strategies used by the more able students to succeed. Given that such remedial students have already suffered loss of esteem from their failure compared with the success of their more able peers, they need a positive approach which emphasizes a small number of powerful general strategies to give more versatile thinking and greater success.

This causes us to focus on the problem of such students and whether it is possible that they can cope with the changes that the more versatile kind of thinking requires. It raises a serious and fundamental problem which requires empirical research:

(Q) Are remedial students capable of benefitting from teaching which focuses on higher level learning strategies in addition to specific mathematical content?

We would suggest that the answer to this question may be in the affirmative, but only if it is done in such a way that the cognitive strain is not increased by trying to teach such concepts and higher level strategies simultaneously. One possible solution is to teach problem-solving strategies in a separate course from those courses teaching mathematical content and procedures.

We hypothesise that several ingredients are necessary to help remedial students consciously come to terms with the nature of higher level mathematical theory, including: 
(1) Revision of procedures to give success and a sound basis for crystallization of these procedures as manipulable mental concepts.

(2) Reflection on these procedures to give them a foundational meaning that gives the students greater confidence in handling them and begin to encapsulate them as concepts.

(3) Explicit extensions of the procedures to see how they should be coordinated, reversed, and mentally manipulated as "chunks" of mathematical code in a more proceptual manner.

(4) Explicit experience and teaching of mathematical thinking processes which gives them confidence in clarifying, formulating and solving mathematical problems.

It should be clear that such remediation cannot be done at the same time as starting the main course, burdening an already overburdened mind with yet more to do. One possibility is to adopt remedial strategies in a constructive confidence-building manner in a foundational course prior to taking further study. Such a course must contain some revision (item (1)) but it must use the experience and encourage maturation of the thinking processes through items (2), (3) and (4). In particular, the mathematical thinking (4) can be a course which concentrates on mathematical processes to give experience, success and enjoyment of mathematics, to teach the students to reflect on how they do mathematics without the expectation of teaching any new content.

It is envisaged that items (1), (2) and (3) may be treated in sequence within a number of different courses, beginning with revision of elementary procedures, wherever possible giving the student the experience which may hope to give new meaning to the concepts being manipulated. For example, algebraic notation and the conventions of arithmetic may be given a more appropriate notation by using a little simple programming using letters as variables (Tall \& Thomas, 1991). In this way an initial meaning can be given to the symbolism which enables it to be manipulated in a coherent way.

Initial experiences might therefore concentrate on:

(a) interpreting information and translating it into an appropriate symbolism,

(b) understanding the conventions of mathematics and the choice of symbolism in different contexts,

(c) being able both to give initial meaning to symbols and also eventually to manipulate them in a routinized and efficient manner.

Once procedures have become routinized, attention can be turned to the more complex actions that cause remedial students difficulties (and which surfaced clearly in the Diagnostic Test): 
(d) coordinating two or more processes,

(e) reversing or undoing a process,

(f) coping with singular cases (exceptional ones which seem not to follow the general pattern and so require special treatment).

Attention needs to be focused on the important idea that so many symbols, e.g. $2+3$, $3 x+4 y,-3$, sine=opposite/hypotenuse, $\mathrm{f}(x), \lim _{n \rightarrow \infty} s_{n}$, etc, represent both a process of calculation and the result of that calculation:

(g) focusing on the fact that same symbolism represents both the process to get a result and the result itself,

(h) crystallizing processes into concepts (by the simple process of meaningfully using the same notation for both).

Knowing that the less able are more likely to want to operate procedurally, the task of focussing on coordinating procedures and manipulating them as concepts needs to be done in contexts where the more powerful methods of operation clearly lead to easier ways of doing the mathematics.

Students need to also be aware of:

(i) using different representations of the same concept (e.g. graphical, numerical, symbolic),

(j) transferring between different representations, to use whichever is easier for a given task.

It cannot be expected that the less able will, by some magical process, be brought to a position where they operate indistinguishably from the more able, desirable though this may be. But, cognisant of the different qualities of thinking processes between those who succeed and those who fail, we hypothesise that a plausible way in which students may become more successful is to become consciously aware of more successful thinking strategies and that this must be done in a context designed to impose less cognitive strain. The implementation of such a remediation strategy is now the subject of ongoing research.

\section{Acknowledgements:}

We like to acknowledge the cooperation and the hard work done by our colleagues of the Diagnostic and Remedial Mathematics Group, Universiti Teknologi Malaysia for implementing and devising the Diagnostic Tests in this paper. 


\section{References}

Bartlett F. C. , 1958: Thinking, George Allen Unwin, London.

Bell F. H. , 1978: Teaching and Learning Mathematics (in Secondary schools), Wm.C.Brown Co. Pub., USA.

Chiarugi I., Fracassina, G. \& Furinghetti F., 1990, "Learning difficulties behind the notion of absolute value", Proceedings of PME 13, Mexico, III 231-238.

Ekenstam A. Af \& Greger K. , 1983: "Some aspects of children's ability to solve mathematical problems", Educational Studies in Mathematics 14, 369-384.

Freudenthal., H., 1983: The Didactical Phenomenology of Mathematics Structures, Dordrecht, Holland; Boston, Reidel.

Gagné R. M., 1970: The Conditions of Learning, Holt Rinehart and Winston Inc., New York, 2nd. edition.

Gray E. \& Tall D. O. , 1991: "Duality, Ambiguity and Flexibility in Successful Mathematical Thinking", Proceedings of PME14, Assisi, II, 72-79.

Greer B. ,1987: "Nonconservation of multiplication and division involving decimals", Journal for Research in Mathematics Education 18 37-45.

Laporan Jawatankuasa Kabinet Mengkaji Perlaksanaan Dasar Pelajaran,1979, pp. 18-20 (in Malay).

Laporan Jawatankuasa Mengkaji Taraf Pelajaran di Sekolah-Sekolah, 1982, p.18 (in Malay).

Markovits Z., Eylon B. \& Bruckheimer M., 1988: "Difficulties Students have with the Function Concept", The Ideas of Algebra, K-12, N.C.T.M. 1988 Yearbook, 43-60.

Newell A. \& Simon H.A.,1972: Human Problem Solving, Prentice Hall, N.J., USA.

Steen L.A.,1989: "Mathematics for a new century", The Australian Mathematics. Teacher, $\mathbf{4 5}$ 19-23.

Tall, D. O. \& Bakar, M. N., 1993: "Students' Mental Prototypes for Functions and Graphs", International Journal of Mathematics Education in Science and Technology, 23 1, $39-50$. 
Tall D. O. \& Thomas M. O. J. ,1991: "Encouraging Versatile Thinking in Algebra Using the Computer", Educational Studies in Mathematics, 22 2, 125-147.

Thurston, W. P., 1990: "Mathematical Education", Notices of the American Mathematical Society 37 7, 844-850.

Vinner S.,1983: "Concept definition, concept image and the notion of function", The International Journal of Mathematical Education in Science and Technology, 14, 293305. 